\title{
Molecular Chain Mail
}

\section{Key words}

anthracenes
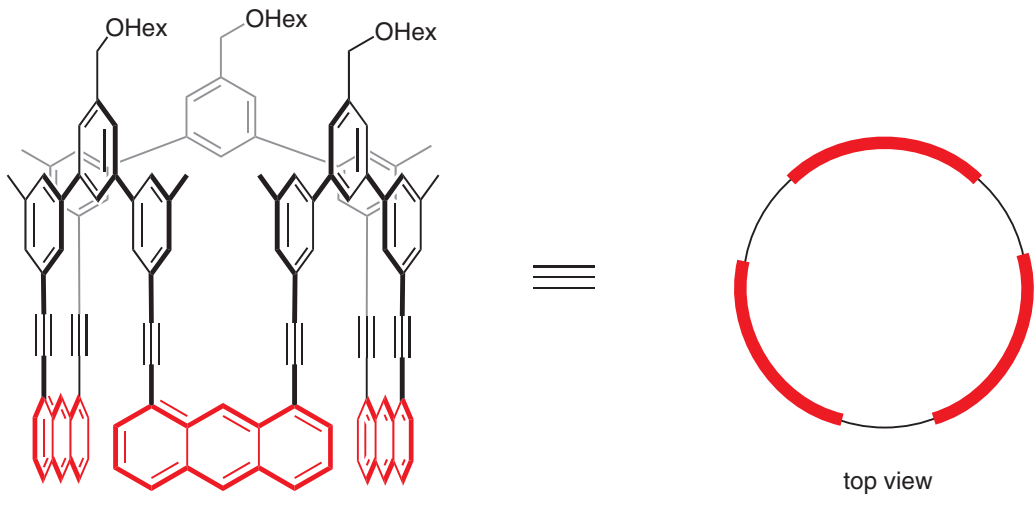

top view

M1
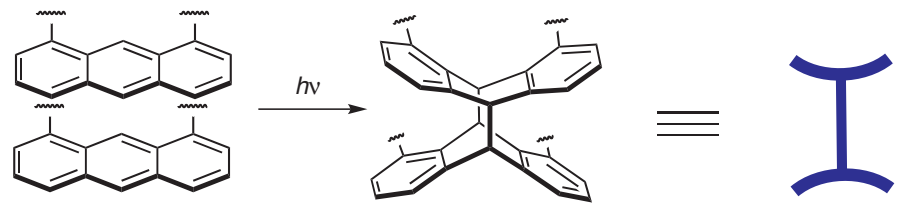

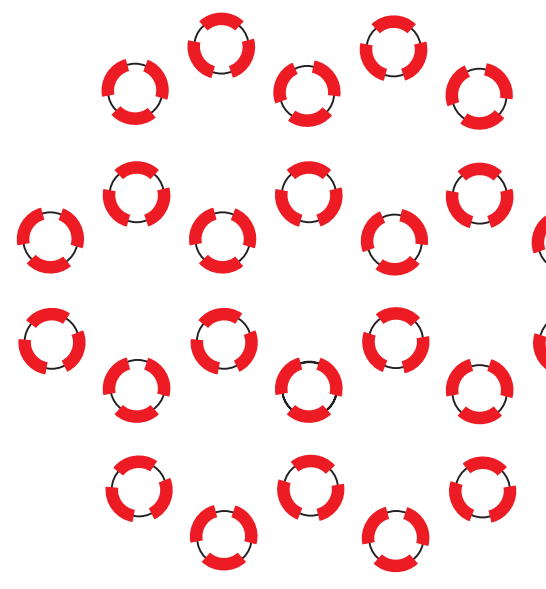

periodic 2D assembly of $\mathbf{M} \mathbf{1}$

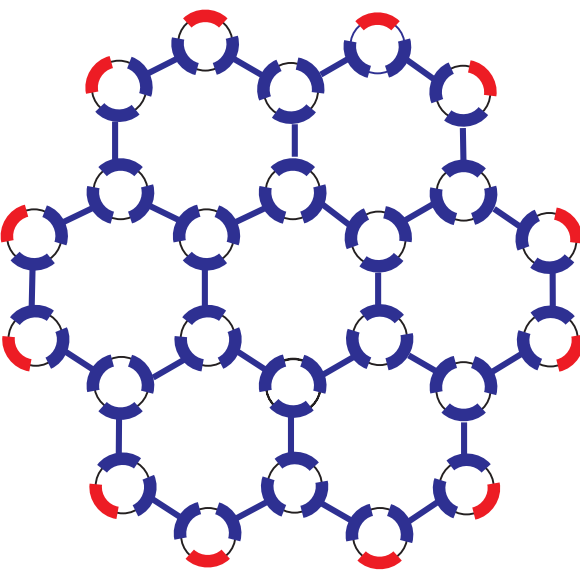

photocrosslinked 2D network of M1 photodimerization

Sonogashira-

Hagihara cross-

coupling

copper-free

Sonogashira crosscoupling

SYNFACTath
Significance: Macrocycle M1, which contains three 1,8-anthrylene units tethered together in $C_{3 v}$ symmetry, was synthesized by iterative palladiumcatalyzed cross-couplings and one copper-free Sonogashira cross-coupling.
Comment: Macrocycle M1 was designed as a potential monomer to yield a 2D polymer network that is a single layer thick. The bottom-up synthesis of such networks has yet to be achieved. Herein, the authors lay the foundations to achieve this goal.

sYNFACTS Contributors: Timothy M. Swager, Trisha L. Andrew 